\title{
OBITUARY
}

\section{John Norris Bahcall 1935-2005}

\author{
Nuclear astrophysicist who uncovered the solar neutrino problem.
}

The philosopher Sir Isaiah Berlin once famously quoted a scrap of Archilochus: "The fox knows many things, but the hedgehog knows one big thing." Astrophysicist John Bahcall would often introduce himself and a colleague to a new acquaintance with the sentence: "I know all about neutrinos, and my friend here knows about everything else in astrophysics."

Such light-hearted self-deprecation was typical of Bahcall, who died on 17 August 2005. But it was inaccurate: Bahcall's scientific interests and expertise ranged from neutrino physics and the structure of the Sun and other stars, to galaxy models, quasars and the intergalactic medium. His more than 600 scientific publications, on an enormous array of subjects, received nearly 20,000 citations. Many established fundamental paradigms in their fields, and others provided the clearest and most comprehensive overview of them.

But Bahcall did, in fact, continually return to one core scientific issue: the solar neutrino problem. He realized very early in his career that we should be able to detect the flux, or stream, of these shadowy fundamental particles as they pass through the Earth after escaping from the centre of the Sun, where they are produced in prodigious numbers. He clearly saw that a definite detection, or non-detection, of these neutrinos would have major implications both for understanding the Sun and for fundamental particle physics. For decades, he encouraged and supported scientists throughout the world in studying this problem and was most successful in his collaboration with Raymond Davis Jr, who ultimately won the Nobel Prize in 2002 for detecting the solar neutrino flux.

It was Bahcall's persistent work that proved definitively that the low flux found by the solar neutrino experiments of Davis and others could not be explained by errors in our model for the Sun. Neutrinos seemed to be missing: either they were not made at the rates required by standard nuclear physics, or they were made but then somehow 'lost' in transit between the Sun and the Earth. The latter explanation - neutrino mixing, in which one type of neutrino changes into another at some rate, and in which the neutrino must have a small but finite mass - is now known to be true, and it is surely due to Bahcall's tenacity and insight that this important and surprising modification to the standard model of particle physics was uncovered.

A fuller idea of his exceptional scientific scope is indicated by the fact that the standard model for a massive black hole surrounded by a cluster of stars is still called the BahcallWolf model; the most widely quoted model for our Galaxy was for decades the BahcallSoneira model; and the most accurate models for the solar interior were those developed by Bahcall, with Roger Ulrich, Marc Pinsonneault and others.

After completing his graduate education at Harvard in 1961 (having started his university education at Louisiana State University on a tennis scholarship), he arrived at the California Institute of Technology (Caltech), working with Willy Fowler and others at the time and place that 'nuclear astrophysics' was invented. There he became engaged with neutrino work and to Neta Assaf (then completing her $\mathrm{PhD}$ at Caltech) - the two constant loves of his life. His first paper from Caltech, a one-page letter to the editor of the Astrophysical Journal, dated 1 December 1962 and entitled "The Solar Neutrino Flux" (authored with Fowler, Icko Iben and Richard Sears), proposed an experiment that might "provide a valuable experimental limit on the effective temperature for neutrino generation in the sun". That paper set the course for a lifetime of research.

The writing of scientific papers was, however, only one of Bahcall's many contributions to world science. He was an educator who changed the nature of postdoctoral training, and a scientific statesman of unusual and beneficent influence. Bahcall moved to the Institute for Advanced Study (IAS) at Princeton in 1968 and soon established that institution as a magnet and model for postdoctoral training. A significant fraction of the world's most distinguished astrophysicists benefited from his tutelage and the intellectually fertile atmosphere that he established there. The eminent British scientist Sir Martin Rees describes himself as fortunate to have been one of the first IAS postdoctoral fellows in astrophysics in 1969. The birthdays of all the fellows and important family events were celebrated. The intellectual atmosphere was intense, and the weekly Tuesday lunches, with John presiding, to which the whole Princeton physics community was invited, were legendary. Bahcall's postdoc programme was the one that astrophysics institutions worldwide emulated. At the IAS, young scientists were selected and recruited in the most exacting manner and then were free to work on whatever they wanted, with whomever they wished.

While maintaining a scientific and educational programme that would have exhausted most, Bahcall also demonstrated extraordinary scientific leadership. He was president of the American Astronomical Association, led

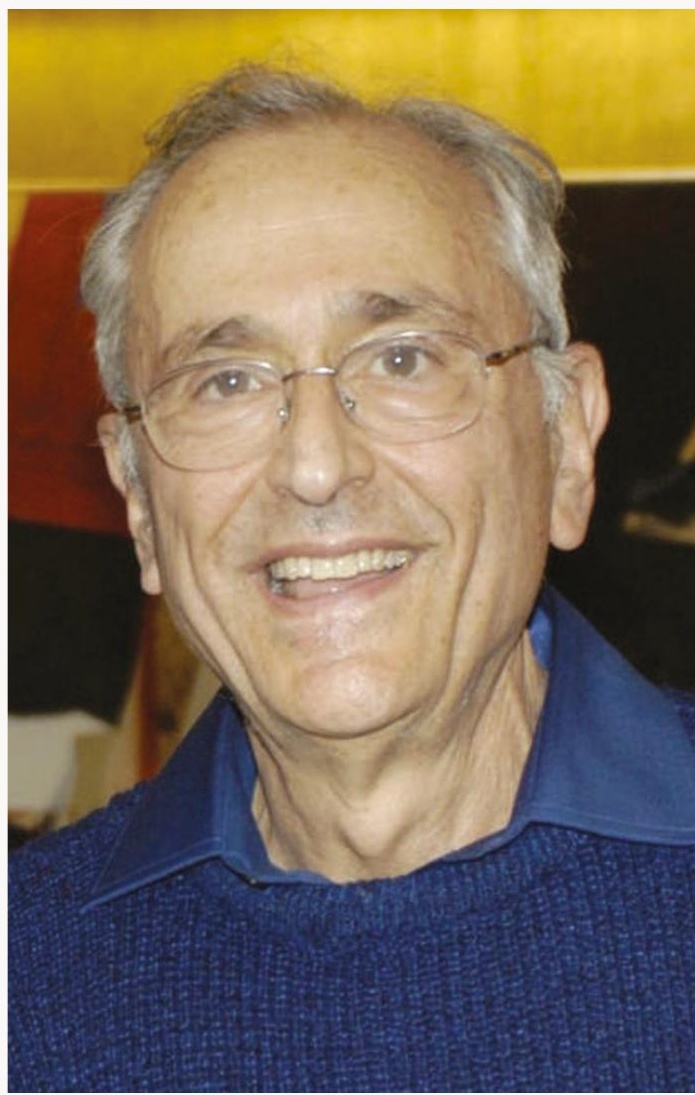

the team that produced the 1990 National Research Council 'Bahcall Report' that set the scientific and instrumental priorities for astrophysics in the United States for a decade, and worked (with Lyman Spitzer Jr) with tireless effectiveness in public and in private to have the Hubble Space Telescope and the Space Telescope Science Institute (STScI) built and maintained as one of the world's pre-eminent scientific facilities.

Neta Bahcall, a distinguished scientist herself, was his love, his best friend and his scientific colleague throughout. She took a leading scientific role at the STScI and wrote over 30 papers with him on subjects ranging from solar neutrinos to binary X-ray sources. They also collaborated in raising three talented children, Safi, Dan and Orli, who are themselves now establishing significant scientific careers.

But no listing of achievements can convey the impression of the man: the wit, the mischievous energy, the passion. Jerry Wasserburg, his old Caltech friend, portrays Bahcall in 1965: "John, running around in white tennis shorts, very sportive and competitive in both creative science and tennis, trying out and enthusiastically arguing every new idea in astrophysics; he was the dynamo of the institute."

\section{Jeremiah P. Ostriker}

Jeremiah P. Ostriker is in the Department of Astrophysical Sciences, Princeton University, Peyton Hall, Ivy Lane, Princeton, New Jersey 08544-1001, USA.

e-mail: ostriker@princeton.edu 